\title{
Dry Composite Electrodes With Carbon Nanotubes Additive for Biopotentials Measurements: ECG Use Case
}

\author{
Dominik Grochala $^{1}$, Marcin Kajor ${ }^{1}$, Paweł Smoleń ${ }^{2}$, Piotr Augustyniak ${ }^{1}$, Krystian Gruszka ${ }^{3}$ \\ ${ }^{1}$ Faculty of Electrical Engineering, Automatics, Computer Science and Biomedical Engineering \\ AGH University of Science and Technology Kraków, Poland \\ ${ }^{2}$ Smart Nanotechnologies S.A. Alwernia, Poland \\ ${ }^{3}$ Department of Cardiology, Interventional Electrocardiology and Arterial Hypertension, \\ Jagiellonian University Medical College, Kraków, Poland
}

\begin{abstract}
Long term monitoring of patients health condition is a useful diagnostic method however it still remains challenging to apply. Prolonged electrocardiographic monitoring and early diagnosis of arrhythmias enables the introduction of appropriate treatment prolonging life expectancy. Nevertheless the market demand is focused on wearable solutions which do not require to stick electrodes to skin. In this research we developed samples of material and evaluated them for suitability in electrophysiological measurements. Favorable conductive properties were achieved by polymer composite containing multi-walled carbon nanotubes and Ag nanoparticles. ECG signals were recorded in limb leads configuration using $M$ TRACE Electrocardiograph device. As a reference standard metal electrodes were used. The examination was conducted in two variants: with and without conductive gel. The signals were then compared and approved by a professional physician as fully diagnostable. Finally, the research revealed that, when comparing to reference electrodes, the signals recorded with designed electrodes in dry conditions featured $27 \%$ higher amplitude and $60 \%$ higher baseline noise whilst in wet conditions (when using conductivegel): $23 \%$ and $55 \%$ respectively. Finally the results showed that designed material features antimicrobial properties (against S. aureus, E. coli).
\end{abstract}

\section{Introduction}

Although electrocardiography (ECG) has been used in medicine since the 19th century, it is still one of the most popular diagnostic methods in many clinical cases. However currently the development of equipment for prolonged ECG monitoring seems to be particularly important. According to the guidelines, it is suggested to use prolonged ECG monitoring in the diagnostic process of bradyarrhythmia or tachyarrhythmia where these disorders occur paroxysmally. The basis for diagnosis and further treatment is then an ECG signal recorded during the pathological episodes[1, 2]. Long term ECG monitoring techniques are also used in the diagnosis of ischemic strokes causes.[3] Ischemic stroke is one of the most common causes of deaths and disabilities in well developed countries [4].

A frequent origin of ischaemic stroke is cardioembolism accompanying atrial fibrillation. Nevertheless up to $40 \%$ of ischaemic strokes are classified as cryptogenic (with no etiology identified after routine diagnostic process). In these cases it is impossible to apply the appropriate secondary prevention [5]. A meta-analysis involving more than 10,000 people showed that in patients with cryptogenic stroke a 3 weeks long ECG monitoring revealed paroxysmal atrial fibrillation in $16.9 \%$ subjects and after 24 months of ECG monitoring in $43 \%$ of patients paroxysmal atrial fibrillation was diagnosed[6]. External or implantable devices are usually used for carrying out a prolonged cardiac monitoring. The implantable ECG loop recorder is aimed at long-term monitoring, and ensures a continuous recording of the signal. However, it features disadvantages like invasive procedures of implantation, possibility of local infections, only one ECG lead or limited battery life [7]. New types of external cardiac monitoring devices are widely researched together with new types of electrodes which would prevent contact hypersensitivity and skin side effects $[8,9]$.

Carbon nanotubes have been a target of scientists throughout the last years. The increased interest of this material can be easily justified by outstanding mechanical and physical properties of these inconspicuous particles. Carbon nanotubes feature Young modulus of 1-5 TPa, high flexibility, high thermal conductivity and metallic or semiconducting character. Thus, combined with a polymer matrix, they allow to obtain composites of extraordinary properties, even in low concentration. Allaoui et al. fabricated multi-wall carbon nanotube/epoxy resin composites based on over-aged hardener and investigated 
mechanical and electrical properties of obtained rubbery material. The percentage of added nanotubes significantly influenced the resulting mechanical characteristics, i.e. the Young modulus and the yield strength have been doubled in case of $1 \%$ addition of the carbon filler compared to the pure polymer matrix. Furthermore, $4 \%$ of the nanoadditive resulted in quadrupled effect. However, these samples were highly viscous and difficult to homogenize. Finally the insulator-to-conductor transition caused by the percolation phenomenon was observed for nanotube concentration between $0.5 \%$ and $1 \%[10]$.

The use of dry electrodes for electrophysiological measurements in wearable applications has been widely justified by both scientific and commercial reports. Probably the most important aspect of such a solution is undoubtedly the electrode-skin impedance. This compound phenomenon can be investigated with the impedance spectroscopy. Bosnjak et al. took advantage of a Schlumberger SI 1260 impedance/gain-phase analyser in conjunction with a tissue sample interface SOLARTRON 1294 to evaluate five different types of dry electrodes which are used in electrocardiography and electroencephalography. The used frequency range was $1 \mathrm{~Hz}-750 \mathrm{kHz}$. The research proved that all electrodes featured an impedance in range between $500 \mathrm{kOhm}$ and $1 \mathrm{MOhm}$ and the multi-pin polyurethane-A electrode revealed the lowest impedance at all investigated frequencies[11]. Chlaihawi et al. developed a new kind of dry electrodes for measuring biopotentials. The composition of MWCNT and PDMS (polydimethylsiloxane) was applied by screen printing into silver-coated PET (polyethylene terephthalate). The ECG waveforms and the measurable skin-electrode impedance were recorded. Obtained results confirmed the high usefulness of this type of electrode, characterized by a conductivity of $938.8 \mathrm{mS} / \mathrm{m}$, with better properties compared to electro-gel electrodes, especially when the patient was in motion.[12] Peng et al. described the method of producing dry electrodes based on MWCNT and PDMS. Samples have been formed into a special shape of protruding tubes. Conducted research indicates the high efficiency of the solution when the patient is moving, but also proves suitability of proposed material in long-term measurements and wearables application. Finally, authors pointed out extreme difficulties they faced during material processing, namely thorough homogenization of the material, for which they used tolueen[13].

The aim of the work was to test the suitability of the developed electrode material in practice. More detailed material characterization analysis and property measurements as well as optimization of the manufacturing process are the subject of ongoing development work[14]. At this stage, the goal was to examine the samples in their target application, using both automated ECG analysis and the opinion of a specialist physician.

\section{Materials and methods}

Commercially available multi-wall carbon nanotubes were used to obtain polymer nanocomposites. Thin multiwall carbon nanotubes NC7000 from Nanocyl are produced via the Catalytic Chemical Vapor Deposition (CCVD) process. Nanotubes have an average diameter of $9.5 \mathrm{~nm}$ and length of $1.5 \mu \mathrm{m}$. Carbon purity in this powder component was at least $90 \%$. The polymer nanocomposite has been synthesized by mixing hydroxy functional polydimethyl siloxane polymer, (vinylmethylsiloxane)dimethylsiloxanevinyl terminated copolymer and hightemperature vulcanizing silicone rubber. Substances reducing the viscosity of the uncrosslinked material were also used in order to obtain the best dispersion of nanoadditive in polymer. To homogenize all components, a laboratory triple roller was used. This device contains three independent shafts moving axially in the opposite phase driven by an electric motor. After thorough mixing, $7.5 \mathrm{wt} \%$ carbon nanotubes were added to the polymer base. After obtaining a homogeneous mixture, a catalyst system was added, which included an inhibitor (3,5-dimethyl-3hexanol) and platinum-based polymerization catalyst. Then, the mixture was deaerated and formed into appropriate shapes between teflon sheets and crosslinked for 30 minutes at 150 degrees Celsius.

In the case of polymers with nanosilver additive (Smart Nanotechnologies S.A.), a special colloid was predispersed into (vinylmethylsiloxane)dimethylsiloxanevinyl terminated copolymer. Microscopic studies revealed that the predominance of obtained silver nanoparticles is spherical in shape and features diameter below $10 \mathrm{~nm}$. The concentration of silver in the final nanocomposite was $150 \mathrm{ppm}$.

The developed material was molded into discs with an embedded mesh made of stainless steel as a contact, and used in such a form during an experimental study as electrodes.

Microbiological tests were carried out to analyze the antimicrobial properties of prepared nanocomposites. Samples containing multi-walled carbon nanotubes with and without nanosilver have been subjected to microbiological tests according to ISO 22196: Plastic Measurement of antibacterial activity on plastic surfaces. In addition, the control was a polystyrene Petri dish. The cover film was sterile Stomacher bags made from $70 \mu \mathrm{m}$ thick polyethylene, from which $4 \mathrm{~cm}^{2}$ squares were cut out. Escherichia coli (ATCC 8739) and Staphylococcus aureus (ATCC 6538) were used as test microorganisms. The inoculum volume was $0.2 \mathrm{~cm}^{3}$ and number of live bacteria in the inoculum for the strains Staphylococcus aureus and Escherichia coli was $7 \cdot 10^{5} \mathrm{CFU} \cdot \mathrm{cm}^{-3}$ for both. The contact time was 24 hours. 
ECG measurements were conducted on one male volunteer during a single study. The subject was in a supine position consistent with the 12-lead rest ECG examination concept. Four limb electrodes with embedded metal plates were used in the form of clamps fixed to the limbs. A set of six metal electrodes with suction cups were placed on the chest surface. The reference configuration was based on electrodes without modification. The measurement of the developed composite electrodes was performed by replacing the lead contacts with composite discs, while standard clips were used to maintain the mechanical pressure on the skin surface. The precordial leads were not replaced with the tested composite material due to the difficulty of firmly fixing of the samples-electrodes

The signals were recorded using the M-TRACE device manufactured by M4Medical Sp. $\mathrm{z}$ o.o. Poland. Electrocardiograph was set in 12-lead AUTO mode with following parameters: Isoline $-1.5 \mathrm{~Hz}$, Low pass filter $35 \mathrm{~Hz}$, Power line filter $50 \mathrm{~Hz}$ and sampling rate of $500 \mathrm{~Hz}$. As a comparable and reference quantitative value, distortion parameter and $\mathrm{R}$ wave amplitude (I lead) was used. Those parameters are available in the auto analysis mode of utilized electrocardiograph. What is more, this functionality is only available for examination conducted with all electrodes. This caused the need to record the signal also with metallic precordial electrodes, while the limb pads were made of developed composite. As the second step in the assessment of the usefulness of obtained signals, the traces were presented to a physician specialist, so that ECG strips can be analyzed on the basis of clinical experience. Physician's task was not to diagnose the particular patient but to share the opinion whether the signal in presented form was sufficient to make a diagnosis and whether it would be consistent with the diagnosis based on the examination using the factory set of electrodes.

\section{Results}

The ECG signals were collected during one examination and the test was repeated four times for each of three configurations, i.e. standard dry electrodes, developed composite electrodes and standard dry electrodes with the addition of conductive gel. Resulting average values of $\mathrm{R}$ wave amplitude and distortion are listed in the Table1. A specialist physician assessed the ECG strips and found them diagnostically useful (Fig. 1)

The antimicrobial properties of designed electrodes were evaluated according to the procedure listed in paragraph 2.1. Results confirm high antimicrobial activity against Gram-negative bacterial strain (Escherichia coli) and Gram-positive (Staphylococcus aureus). In case of both tested bacterial strains, the reduction reached $100 \%$. The obtained results confirm the huge antimicrobial potential of synthesized nanocomposite, and thus prove a wide range of their possible applications, especially in the medical field.

Table 1. Averaged results of the distortion measured with the electrocardiograph and the amplitude of the $\mathrm{R}$ wave

\begin{tabular}{ccc}
\hline Parameter: & $\begin{array}{c}\mathbf{R}(\mathrm{I}) \text { wave } \\
\text { amplitude } \\
{[\mu \mathrm{V}]}\end{array}$ & $\begin{array}{c}\text { Distortion } \\
{[\mu \mathrm{V}]:}\end{array}$ \\
\hline Dry metal & 1067.5 & 5.25 \\
Metal + gel & 1101.3 & 5.5 \\
Composite & $\mathbf{1 3 5 5 . 0}$ & $\mathbf{8 . 5}$ \\
& & \\
Ratios[\%] & $\mathbf{+ 2 6 . 9}$ & $\mathbf{+ 6 1 . 9}$ \\
& $\mathbf{+ 2 3 . 0}$ & $\mathbf{+ 5 4 . 6}$ \\
\hline
\end{tabular}

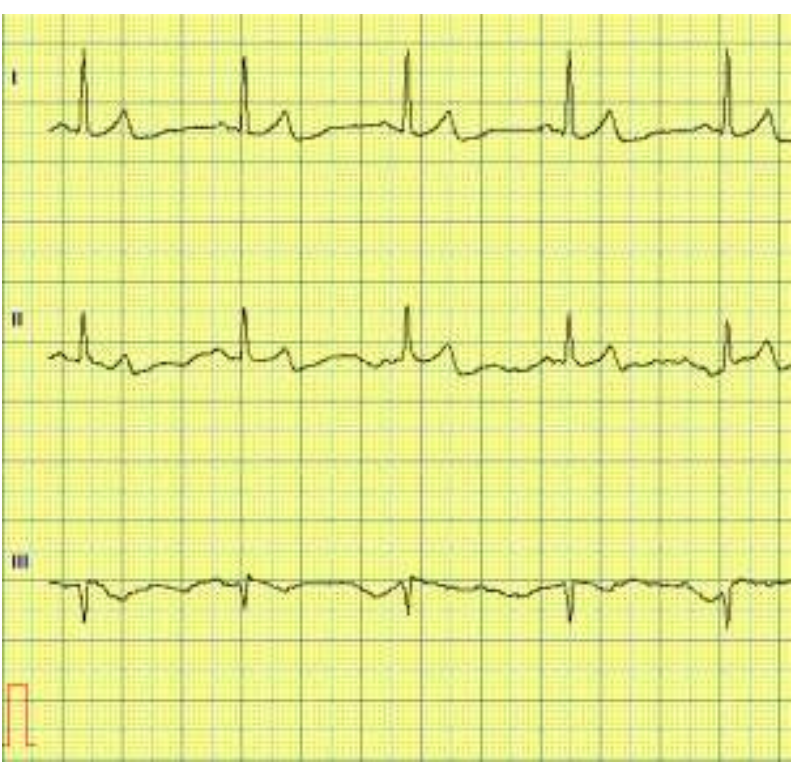

Figure 1. An example waveform obtained with developed composite electrodes.

\section{Discussion and conclusions}

In this paper we described a process of fabricating novel dry composite electrodes for electrophysiological measurements. Carbon nanotubes fraction allowed to obtain an impedance required for ECG applications and the silicone rubber matrix ensured satisfying mechanical characteristics. Microbiological tests proved that the silver nanoadditive ensured high antimicrobial properties of the resulting samples. Finally we evaluated the ECG signals acquired with the use of designed electrodes and compared them with measurements recorded with 2 other electrode types. The signal obtained from developed composite electrodes turned out to be more prone to baseline disturbances. 
We observed that on the interface between proposed electrodes and the skin, there are unfavorable phenomena that deteriorate electrical contact. These include a series of capacitances manifested by slowly alternating disturbances of large amplitude. Despite the aforementioned issues, received signals indicate their diagnostic usefulness both in terms of measurable quantitative parameters and the opinion of a specialist.

Proposed new dry electrodes may become a part of comfortable wearable external devices dedicated to long term ECG monitoring. These electrodes will be easy to use and may contribute to increasing the quality of life of patients who require prolonged ECG monitoring, as well as promoting high level of patient compliance. As a result proposed solution may lead to better efficiency in the diagnosis of arrhythmias.

\section{Acknowledgments}

This research was partly funded by AGH University of Science and Technology in 2020 as research project No. 16.16.120.773 and was carried out in cooperation with Smart Nanotechnologies S.A.

\section{References}

[1] M. Brignole et al., "2018 ESC Guidelines for the diagnosis and management of syncope," European heart journal, vol. 39, no. 21, pp. 18831948, 2018.

[2] J. Brugada et al., "2019 ESC Guidelines for the management of patients with supraventricular tachycardiaThe Task Force for the management of patients with supraventricular tachycardia of the European Society of Cardiology (ESC) Developed in collaboration with the Association for European Paediatric and Congenital Cardiology (AEPC)," European heart journal, 2020.

[3] A. Culebras, S. R. Messé, S. Chaturvedi, C. S. Kase, and G. Gronseth, "Summary of evidencebased guideline update: prevention of stroke in nonvalvular atrial fibrillation: report of the Guideline Development Subcommittee of the American Academy of Neurology," Neurology, vol. 82, no. 8, pp. 716-724, 2014.

[4] S. S. Virani et al., "Heart disease and stroke statistics-2020 update: a report from the American Heart Association," Circulation, pp. E139-E596, 2020.
[5] L. Li et al., "Incidence, outcome, risk factors, and long-term prognosis of cryptogenic transient ischaemic attack and ischaemic stroke: a population-based study," The Lancet Neurology, vol. 14, no. 9, pp. 903-913, 2015.

[6] J. E. Dalen and J. S. Alpert, "Silent atrial fibrillation and cryptogenic strokes," The American Journal of Medicine, vol. 130, no. 3, pp. 264-267, 2017.

[7] A. Bisignani, S. De Bonis, L. Mancuso, G. Ceravolo, and G. Bisignani, "Implantable loop recorder in clinical practice," Journal of arrhythmia, vol. 35, no. 1, pp. 25-32, 2019.

[8] L. Stingeni et al., "The role of acrylic acid impurity as a sensitizing component in electrocardiogram electrodes," Contact Dermatitis, vol. 73, no. 1, pp. 44-48, 2015.

[9] C. Lou et al., "Flexible graphene electrodes for prolonged dynamic ECG monitoring," Sensors, vol. 16, no. 11, p. 1833, 2016.

[10] A. Allaoui, S. Bai, H.-M. Cheng, and J. Bai, "Mechanical and electrical properties of a MWNT/epoxy composite," Composites science and technology, vol. 62, no. 15, pp. 1993-1998, 2002.

[11] A. Bosnjak, P. Linares, J. McLaughlin, and O. J. Escalona, "Characterizing dry electrodes impedance by parametric modeling for arm wearable long-term cardiac rhythm monitoring," in 2017 Computing in Cardiology (CinC), 2017: IEEE, pp. 1-4.

[12] A. A. Chlaihawi, B. B. Narakathu, S. Emamian, B. J. Bazuin, and M. Z. Atashbar, "Development of printed and flexible dry ECG electrodes," Sensing and bio-sensing research, vol. 20, pp. 915, 2018.

[13] H.-L. Peng et al., "Flexible dry electrode based on carbon nanotube/polymer hybrid micropillars for biopotential recording," Sensors and Actuators A: Physical, vol. 235, pp. 48-56, 2015.

[14] D. Grochala, M. Kajor, P. Smoleń, and E. Stodolak-Zych, "Preliminary investigations on silicone resin composites with carbon filler for dry electrodes application," Engineering of Biomaterials, vol. 22, no. 149, 2019.

Address for correspondence:

Dominik Grochala

al. Mickiewicza 30 30-059 Kraków Poland

grochla@agh.edu.pl 